\title{
La dictature des colonels en Grèce, 1967-1974
}

Les enjeux géostratégiques

The colonel's dictatorship in Greece, 1967-1974, geostrategical stakes

\section{Corinne Talon}

\section{OpenEdition}

\section{Journals}

Édition électronique

URL : https://journals.openedition.org/ceb/1105

DOI : $10.4000 /$ ceb. 1105

ISSN : 2261-4184

Éditeur

INALCO

Édition imprimée

Date de publication : 30 mars 2011

Pagination : 379-402

ISBN : 978-2-85831-189-7

ISSN : 0290-7402

Référence électronique

Corinne Talon, «La dictature des colonels en Grèce, 1967-1974 », Cahiers balkaniques [En ligne], 38-39 |

2011, mis en ligne le 11 décembre 2012, consulté le 06 juillet 2021. URL : http://

journals.openedition.org/ceb/1105; DOI : https://doi.org/10.4000/ceb.1105

Ce document a été généré automatiquement le 6 juillet 2021.

Cahiers balkaniques est mis à disposition selon les termes de la Licence Creative Commons Attribution - Pas d'Utilisation Commerciale 4.0 International. 


\title{
La dictature des colonels en Grèce, 1967-1974
}

\author{
Les enjeux géostratégiques \\ The colonel's dictatorship in Greece, 1967-1974, geostrategical stakes
}

\section{Corinne Talon}

1 Cet article s'appuie sur une thèse de doctorat : «L'image de la dictature des colonels en Grèce, à travers la presse d'opinion française: Le Monde, Le Figaro et L'Humanité, 1967-1974 ", soutenue par l'auteur en décembre 2009, à l'Université Montpellier III Paul Valéry. En l'absence de toute recherche en français sur ce sujet, il s'agissait de présenter la plus large interprétation possible de ce régime et d'en montrer les différents points de vue et analyses, à travers l'étude de trois quotidiens nationaux français, de sensibilités politiques différentes ou censées l'être.

2 Le 21 avril 1967, à deux heures cinq du matin, les chars de l'armée grecque envahirent le cœur d'Athènes. Des militaires, parmi lesquels trois hommes: le colonel Georges Papadopoulos, le colonel Nicolaos Makarezos et le général Stylianos Pattakos, apparurent rapidement à la tête du pouvoir et imposèrent en Grèce, ce qui est communément appelé : «la dictature des colonels». Les articles de la constitution relatifs aux droits de l'homme furent immédiatement suspendus et les principaux responsables politiques du pays (membres du gouvernement et de l'opposition) furent arrêtés. Les trois militaires justifièrent leur action par l'imminence d'une menace communiste.

3 La dictature des colonels s'est instaurée et déroulée dans un contexte de guerre froide. Membre de l'OTAN depuis 1951, la Grèce appartenait au «bloc de l'Ouest » et se situait à proximité des voisins de « l'Est »: Bulgarie, Albanie et Yougoslavie. Durant la dictature, la Méditerranée orientale connut différentes tensions et conflits, dont la guerre des Six Jours en 1967 et celle du Kippour en 1973, tandis que l'île de Chypre, République indépendante depuis 1960 - mais qui accueillait des bases militaires britanniques et des troupes grecques, turques et onusiennes - suscitait des convoitises diverses et notamment celle des Grecs favorables à l'Enosis (le rattachement de l'île à la Grèce). 
Cette situation entraîna des tensions avec la Turquie. Ces tensions, entre deux membres de l'OTAN, ne pouvaient manquer de faire réagir les États-Unis, et ceci d'autant plus que Chypre se situait à proximité d'une zone d'opposition entre les deux Grands : le Proche-Orient. La situation chypriote suscitait d'ailleurs l'inquiétude de l'URSS, qui était déterminée à défendre l'indépendance de l'île, face aux États-Unis, dont l'influence croissait dans cette partie du monde.

Cet article présente les interprétations et les analyses des trois quotidiens nationaux français sur le rôle de ces enjeux géostratégiques, le rôle et/ou l'influence des ÉtatsUnis, mais aussi celui de Chypre, dans l'évolution politique grecque, à savoir : la mise en place de la dictature, sa pérennité et sa chute. Cet article s'articule autour de trois périodes: 1967, année de la mise en place de la dictature et celle d'un danger communiste ? La deuxième : 1968-1973 s'attache au rôle des États-Unis dans le maintien du régime, mais aussi dans ses évolutions. Enfin, 1974, année de la chute de la dictature, chute, qui semble liée, à la situation chypriote et au rôle des États-Unis.

\section{7 : Parer la « menace » communiste ?}

Des élections législatives anticipées étaient prévues fin mai 1967 en Grèce, mais elles intervenaient dans un contexte politique complexe ${ }^{1}$.

Depuis 1965, et la découverte du complot «ASPIDA $»^{2}$ dans l'armée, un complot de gauche qui aurait eu pour but de renverser la monarchie et qui incriminait des officiers, mais aussi Andréas Papandréou, député, et fils de Georges, Premier ministre et dirigeant de l'Union du centre, la situation politique grecque apparaissait extrêmement tendue. La découverte de ce complot entraîna la démission de Georges Papandréou dont le parti était largement majoritaire à la Chambre des députés. Après cette démission, les gouvernements se succédèrent sans réelle majorité. Cependant, une partie des députés de l'Union du centre abandonnèrent leur parti et permirent ainsi la formation de gouvernement «centriste» avec le soutien de l'ERE (parti de droite). En décembre 1966, pour sortir de la crise, «un accord» fut passé entre M. Georges Papandréou et M. Panayotis Canellopoulos, dirigeant de l'ERE, pour envisager l'organisation de nouvelles élections. Le gouvernement Paraskevopoulos fut nommé et chargé de préparer ces élections anticipées. Mais des désaccords entre les deux partis entraînèrent sa démission. Le roi nomma $M$. Canellopoulos, chef $d u$ gouvernement le 3 avril 1967. Ne disposant pas de majorité, il annonça la dissolution de la chambre et fixa les nouvelles élections au 28 mai $1967^{3}$. Mais le coup d'État intervint. Les colonels justifièrent leur coup d'État par l'existence d'une menace communiste, d'un coup de force, qui serait survenu à l'occasion du premier discours électoral de Georges Papandréou à Salonique le 23 avril 1967.

\section{Une menace communiste?}

7 Les trois quotidiens reprennent les déclarations officielles, selon lesquelles « de larges infiltrations communistes venant de l'extérieur étaient assemblées à Salonique, dans le but de déclencher un coup de force [...]le 23 avril [...]». Pour Le Monde et L'Humanité, il n'y a jamais eu de menace communiste et, le coup d'État a été préparé: «[...] les militaires ont pris le pouvoir [...] selon un «plan» préparé à l'avance [...] » $»^{4}$ «Il est inutile de préciser que jamais la moindre preuve d'un « complot communiste » n'a pu 
être fournie [...] $»^{5}$. Pour Le Monde, les déclarations officielles sont fausses: «[...]l'intervention a été motivée par la « dégradation» des mœurs et des institutions confirmant ainsi l'impression que l'ambition [...] allait au-delà du but invoqué au début «éviter les effusions de sang à Salonique " ${ }^{6}$. "[...] Faut-il en déduire que le « coup d'État avait été préparé de longue date ? C'est ce que l'on pense généralement [...] $\gg^{7}$.

Pour Le Monde et L'Humanité, l'objectif immédiat du coup d'État était d'empêcher les élections dont l'Union du centre devait sortir vainqueur. Pour le premier: «[...] La consultation risquait fort [...] de confirmer la victoire électorale de l'Union du centre $»^{8}$. Pour le second : «[...] les observateurs les plus divers s'accordent à reconnaitre [...] que le verdict populaire aurait confirmé et, sans doute, accentué la défaite subie par le parti de droite en février 1964 [...] "9. Il s'agit d'une « dictature absurde et criminelle qui s'est imposée par peur des élections $»^{10}$.

9 La position du Figaro est, en revanche, totalement différente. Pour le journal, l'attitude de Georges Papandréou, «l'affaire ASPIDA » et les désordres liés à celle-ci expliquent le coup d'État: "Devant la menace d'une anarchie complète, l'armée a décidé d'intervenir [...] $»^{11}$. La menace communiste semble donc réelle pour le journal et le coup d'État paraît justifié sinon nécessaire. D'une part, le journal reprend sans les commenter les propos d'un des putschistes: «il existait un plan visant à une action subversive ainsi que les moyens de la réaliser [...] $\gg^{12}$. D'autre part, le journal estime qu'il existait un risque de guerre civile lié aux élections: "[la] pauvreté [de la Grèce] [...] rend malaisé l'exercice d'une démocratie à l'occidentale. Le penchant au désordre y est constant [...] le péril était imminent [...] les élections prévues [...] débouchaient normalement sur la guerre civile [...] dans l'hypothèse la plus probable [...] celle de la victoire de la coalition des centristes [...] la démocratie populaire d'obédience orientale se serait heurtée à une opposition [...] dans le cas d'une victoire des modérés [...] la gauche était décidée à contester [...] $»^{13}$. Le danger communiste et les conséquences de celui-ci sont les plus importants pour le journal: " Une Grèce de « Front populaire » aurait à coup sûr quitté l'alliance occidentale [...] tel était le programme avoué des coalisés de gauche [...] cela aurait été « un coup » presque mortel pour l'OTAN en Méditerranée [...] ». Ainsi, grâce ( ?) au putsch du 21 avril : « [...] guerre civile et renversement des alliances [ont] été épargné à la Grèce [...] » ${ }^{14}$. Car, pour le journal : «La Grèce n'est environnée que d'adversaires $[. . .]^{15}$ ». Ce journal est le seul, tout au long de la période, à évoquer la thèse du danger communiste. En effet, et, malgré les dénégations officielles du colonel Papadopoulos faîtes en mars 1969, à Alexandropolis, le journal évoque encore cette menace en 1971 et en $1972:{ }^{16}:$ «[...] Des déclarations officielles ont depuis été faites assurant que ce danger était désormais complètement écarté ». Tout est dans le mot "désormais ». Enfin, citant un Grec en $1972^{17}$ le journal publie : «Pour nous le problème n'était pas de briser la démocratie, mais justement de l'établir, et de la protéger contre la menace communiste, qui sous couvert [de] l'E.D.A revenait en force et risquait d'amener la guerre civile ». Pour Le Figaro, et lui seul, la dictature endiguait une menace communiste et un renversement des alliances au profit des Soviétiques. Quel est alors le rôle des États-Unis dans ce coup d'État? 


\section{Responsabilité des États-Unis dans le coup d'État ?}

10 La position du Figaro semble paradoxale sur ce sujet. D'une part, le journal est le seul à défendre la thèse gouvernementale du complot communiste. D'autre part, à la veille du coup d'État, le journal publie : «A la suite d'un article du New York Times, des rumeurs annonçant l'établissement d'une dictature en Grèce indignent profondément l'opinion grecque [...] de pareils propos [...] laisseraient entendre que les Américains prévoient une dictature en Grèce [...] $»^{18}$. En revanche, après le coup d'État, les États-Unis n'apparaissent en rien responsables pour le journal. Celui-ci n'évoque pas les États-Unis au moment du coup d'État, ni dans les jours qui suivent, et, il précise même, en publiant une dépêche $\mathrm{AFP}^{19}$ que : «Les États-Unis reconsidèrent leur programme d'aide ». Cette position n'est toutefois pas celle des deux autres journaux.

11 Pour L'Humanité, les États-Unis sont responsables du coup d'État. «Jamais le roi et la junte dont les liens avec les services secrets sont notoires n'auraient pu prendre une telle décision sans avoir le feu vert de Washington ${ }^{20}$. En effet: "sans l'OTAN qui contrôle les huit - dixième de l'armée grecque le putsch ne pouvait se faire [...] $»^{21}$, "Sans l'essence américaine [...] les avions et les chars ne pourraient pas longtemps imposer au peuple grec un régime qu'il réprouve $»^{22}$. Pour le journal, le coup d'État résulte de l'application d'un plan américain : «[...] préparé sous l'égide de l'OTAN et des services secrets US [...] [ce plan] Prométhée préparé en cas de guerre avec un pays voisin [...] prévoyait l'arrestation de dirigeants de gauche afin de prévenir une subversion [...] $»^{23}$. Jacques Duclos ${ }^{24}$ est, on ne peut plus clair : « Le coup d'État fomenté par les milieux les plus agressifs de l'impérialisme américain, en accord avec les pires réactionnaires grecs et avec la complicité de la cour [...] est un des éléments de la "stratégie globale » des impérialistes américains [...] ${ }^{25}$. Les enjeux représentés par la Grèce en Méditerranée sont exposés : «Le 12 décembre 1947 [...] [Truman] annonçait son intention de faire de la Grèce, un appendice de l'économie américaine, un arsenal et une base d'agression contre le camp socialiste [...] elle a des frontières communes avec trois pays socialistes [...]».

La position du journal Le Monde est plus ambiguë. En effet, à la veille du coup d'État, et contrairement au Figaro, le journal signalait que: «[...] la menace d'une dictature semble écartée, Washington se [montre] ouvertement hostile à une pareille solution. Il est donc pratiquement acquis que le pays va aux élections $»^{26}$. Cette dernière remarque ne manque pas de souligner le rôle visiblement essentiel des Américains dans la situation politique grecque et le peu d'indépendance de celle-ci ! Mais, pour le journal, et après le coup d'État, les États-Unis ne semblent pas responsables de celui-ci. Il n'y a aucune preuve concernant le rôle des Américains et l'application du plan Prométhée : « un ensemble de mesures, prévues par les services de l'OTAN pour déjouer une éventuelle subversion communiste [...] Mais rien n'est venu confirmer cette hypothèse [...] $»^{27}$. En revanche, pour le journal, les États-Unis ne se sont pas opposés au coup d'État et semblent s'en accommoder : «L'armée grecque entièrement dépendante des fournitures américaines ne paraît pas se heurter à des difficultés de ravitaillement ${ }^{28}$. Il en est de même du régime qui : «[...] ne semble pas rencontrer l'hostilité [des Américains] [...] ». Car, pour Le Monde : « [...] la Grèce est un élément non négligeable dans le dispositif de l'OTAN [...] s'ils furent surpris par le coup d'État, les [États-Unis] n'ont rien voulu tenter pour empêcher sa réussite [...] $»^{29}$. Toutefois, cette position n'est pas celle des opposants à la dictature qui s'expriment dans le journal. 
Richard Someritis ${ }^{30}$ précise, en exemple : «Les putschistes [ont] bénéficié du «feu vert », de l'aide et des « conseils » des agents américains [...] ».

Les positions et analyses des trois journaux sur le rôle des États-Unis semblent donc claires. Toutefois, le 13 décembre 1967, le roi, qui resta en place malgré la dictature, tenta un contrecoup d'État qui échoua et l'amena à s'exiler à Rome. Si Le Monde n'évoque pas le rôle des États-Unis dans cette affaire, L'Humanité et Le Figaro jugent que le roi a été aidé par eux.

Pour Le Figaro: "Ce n'est un secret pour personne [...] Washington a sans nul doute approuvé le roi dans ses projets [...] Le Président [Johnson] l'encouragea très vivement d'une manière ou d'une autre afin de rétablir en Grèce un régime démocratique [...] $»^{31}$. Pour L'Humanité: "La responsabilité des États-Unis est très engagée dans cette affaire [...]. Les États-Unis [...] ont donné le feu vert à l'entreprise [...]. Le discrédit mondial du régime des colonels rejaillissait sur Washington d'où les encouragements à Constantin [...] et les facilités [...] [sur] la base de l'OTAN [...] $»^{32}$.

Ainsi, en 1967, les trois journaux présentent des points de vue différents sur l'existence d'une menace communiste et le rôle des États-Unis dans la mise en place de dictature. La menace communiste avérée dans Le Figaro est un prétexte pour Le Monde et L'Humanité. Pour ce dernier, les États-Unis sont responsables du coup d'État. Pour Le Monde, ils ne sont pas responsables, mais ils ne l'ont pas empêché et s'en accommodent. Le Figaro estime qu'ils n'y sont pas mêlés. Toutefois, le journal semble évoluer dans sa position durant l'année 1967, et considérer comme les deux autres journaux que les États-Unis ne sont pas indifférents au sort de la Grèce.

\section{La « protection américaine » (1968-1973)}

16 Si en mai 1967, les États-Unis décidèrent de suspendre leurs livraisons d'armes à la Grèce, cet embargo ne fut pas réellement appliqué. En effet, à la fin 1968, en raison de l'intervention soviétique en Tchécoslovaquie, le président Nixon décida de lever l'embargo et les États-Unis livrèrent, pour 28 millions de dollars d'armes lourdes, à la Grèce. L'embargo prit officiellement fin en septembre 1969. En février 1972, le port du Pirée devint un des ports de la $\mathrm{VI}^{\mathrm{e}}$ flotte américaine en Méditerranée et, en mars, Washington annonça une aide militaire à Athènes.

17 Il s'agit ici de présenter les analyses des trois quotidiens sur le soutien des États-Unis au régime des colonels entre 1968 et 1973, mais aussi de présenter leurs analyses sur le maintien du régime, sa pérennité, grâce aux États-Unis.

\section{Un régime soutenu par les États-Unis pour des raisons stratégiques}

18 Ce point de vue est défendu par les trois quotidiens. La position du Figaro est en revanche plus ambiguë que celle du Monde et de L'Humanité.

\section{Le Monde et L'Humanité : un régime soutenu par les États-Unis}

Pour Le Monde, l'évolution du régime et son sort dépendent des États-Unis : «Pour des raisons stratégiques, politiques et économiques, le soutien américain [...] ne s'est jamais démenti depuis [...] le 21 avril 1967 [...]. » ${ }^{33}$. En effet, « La vraie force de la junte, c'est la 
position géostratégique de la Grèce dans le système de défense du monde occidental. Pour les États-Unis, une telle position n'a pas de prix $»^{34}$.

Les enjeux géostratégiques sont clairement définis: «l'opposition grecque a presque abandonné la théorie du coup de téléphone de Nixon qui pourrait abattre le régime [...], car elle sait que [...] Washington veut avant tout que l'Occident militaire ne s'arrête pas demain à l'Italie [...] $»^{35}$, « la reprise des livraisons [...] d'armement lourd [...] «les facilités portuaires » obtenues par la VIe flotte au Pirée [...] [les] bases militaires dont dispose l'OTAN [...] la décision [...] de M. Nixon de vendre trente-six chasseurs bombardiers [...] témoignent de l'importance que confère Washington à l'allié grec [...] » ${ }^{36}$. En effet : «La présence russe en Méditerranée orientale, la persistance du conflit israélo-arabe, le désengagement de Malte, l'ombre sur Chypre, la confusion en Turquie [...] l'éventualité de voir les Russes intervenir dans les affaires yougoslaves et avoir ainsi accès à l'Adriatique font qu'aux yeux des Américains [...] la Grèce devient une pièce maîtresse sur l'échiquier [international] [...]. Elle permet aux Américains d'empêcher l'encerclement amorcé par le Sud et, mieux encore, de se placer au seuil du monde communiste $[. ..] »^{37}$.

Cette situation n'empêchait pas selon le journal une certaine opposition : « Toutefois, le conseil de l'OTAN comprend neuf États européens dont l'hostilité au régime d'Athènes est confirmée. Cette opposition latente, conjuguée à celle [...] du Congrès et de l'opinion obligera-t-elle le gouvernement américain à tempérer son aide au régime grec ou à abandonner celui-ci à son sort? $»^{38}$.

L'Humanité partage cette position, mais le journal est plus critique : «Selon le «NewYork Times", Washington craint pour ses intérêts stratégiques en Méditerranée. Le changement de régime en Libye [...] le conflit du Moyen-Orient, font des colonels fascistes des "alliés indispensables » $»^{39}$. Le journal dénonce la politique stratégique américaine: "Un arrêt des livraisons d'armes aux colonels, a déclaré le sénateur démocrate Dodd, risquerait : "d'affaiblir gravement l'OTAN et de saper l'ensemble de notre position défensive dans cette zone méditerranéenne [...] $\gg^{40}$. Quant au choix américain du port du Pirée, il entraîne ce commentaire : «Le Pirée va devenir la plus importante base navale américaine [...]. Jamais l'implantation n'avait été aussi étendue que sous les colonels [...]. L'OTAN avait déjà une base en Crête [...] la VI flotte qui avait déjà une base pour ses sous-marins à Corfou, va débarquer au Pirée. Il n'est pas difficile de deviner quels gages les colonels ont reçus en échange: le soutien dont ils ont bénéficié [...] va être renforcé [...] ».Pour L'Humanité, la présence américaine dans cette région constitue une menace pour la paix : «Au cours de la seule période de Noël, 32 navires de guerre américains ont jeté l'ancre près d'Athènes [...] Être solidaire du peuple grec, c'est aussi dénoncer les dangereuses intrigues du Pentagone dans cette région chaude $»^{41}$. Si L'Humanité n'hésite pas, pour des raisons idéologiques évidentes, à critiquer l'attitude des États-Unis. En revanche, dans Le Monde, l'attitude des États-Unis est dénoncée par l'opposition à la dictature : «[...] la Grèce [aurait] besoin d'un régime fort, seul garant des bases américaines en Grèce, aujourd'hui indispensable pour tenir en échec l'expansion soviétique en Méditerranée ». Cette thèse est : «fallacieuse [...] désuète [...] à l'heure des fusées intercontinentales », car elle s'appuie sur l'idée que « les bases américaines sont menacées par une démocratie grecque véritable du fait que celle-ci serait fatalement dominée par l'extrême- gauche ». Or, celle-ci a été : « saignée à blanc pendant la guerre civile [...] décimée par la suite ». 


\section{Le Figaro : un régime nécessaire aux États-Unis}

Le journal expose les événements qui constituent pour le régime des «coups de chance » et qui le rendent nécessaire aux États-Unis. ${ }^{42}$ Il s'agit de : « La guerre des Six Jours [...] [cette guerre en juin 1967] a fait disparaître les colonels, de la scène, au moment où les feux de la rampe menaçaient de les consumer [...] l'invasion de la Tchécoslovaquie [...] les colonels affichent au passage un petit air de triomphe : n'ontils pas été les premiers, un an plus tôt, à annoncer que le communisme représentait au sein même de l'Europe une redoutable menace ? [...] L'OTAN recense les bataillons [...] La situation providentielle de la Grèce dans cette région du monde, au moment où l'on craint que le bloc soviétique n'étende sa "normalisation " à la Roumanie, voire à la Yougoslavie ${ }^{43}$, vaut aux colonels, les premiers sourires étrangers de leur aventure [...] l'élection de Richard Nixon ${ }^{44}[. .$.$] le nombre de bâtiments soviétiques qui franchissent le$ Bosphore augmente. La pénétration du pavillon rouge en Méditerranée devient inquiétante [...], la révolution libyenne [...] et la perte de la base de Wheelus field ${ }^{45}$ et [...] du potentiel énergétique [...] les colonels ne s'y sont pas trompés [...] [pour eux] la défense de l'Occident se déplace désormais sur les bases américaines d'Espagne, d'Italie et de Grèce. La Grèce prend une importance accrue pour la défense des pays occidentaux $»^{46}$. Pour Le Figaro, Les États-Unis constituent "un parapluie $»^{47}$. "Les Américains ne sont sans doute pas aimés sentimentalement comme les Français le sont [...], mais ils sont proches et nécessaires : nécessaires contre la pesante menace slave, comme la Grèce, verrou de la Méditerranée orientale est, avec la Turquie, nécessaire aux Américains. La même armée qui est au pouvoir en Grèce est un des piliers de l'OTAN [...]. Sans elle, la Grèce devient satellite, et la Méditerranée devient russe $[. ..] »^{48}$. Si les enjeux géostratégiques représentés par la Grèce en Méditerranée expliquent la présence américaine selon Le Figaro, en revanche, ils expliquent le soutien des États-Unis au régime dans Le Monde et L'Humanité, mais ces deux journaux vont plus loin.

\section{Le Monde et L'Humanité : un régime qui se maintient grâce aux EU}

Pour Le Monde : «Les rapports gréco-américains [se sont] notablement renforcés depuis le coup d'État du 21 avril 1967 [...] appui militaire, économique et politique des ÉtatsUnis [...] le régime ne parait nullement menacé [...]. La junte serait depuis longtemps renversée si elle ne bénéficiait pas de l'aide américaine " $^{49}$. "Indéniablement les Américains sont partout [...] grosses sociétés (Litton ${ }^{50}$, Ford), les investissements américains [...] et les grandes banques ouvrent de nouvelles succursales [...] À cette aide indirecte s'ajouterait un soutien financier impossible à évaluer [...] pas inférieur à 200 millions de dollars [...] Il manquait une somme de cet ordre au gouvernement pour enrayer la crise économique dans les villes et soutenir le secteur agricole $[. ..] »^{51}$. Le soutien des États-Unis est également affirmé par les opposants dans le journal. Citant le New York Times du 10 septembre 1967, Mario Ploritis ${ }^{52}$ écrit: "Les diplomates américains et les agents de la CIA jouent souvent un rôle aussi important que les hommes politiques grecs [...]». Pour L'Humanité: "Sans l'appui de Washington, la dictature des colonels n'aurait pu se maintenir, pendant ces quatre années $\aleph^{53}$. 


\section{Intervention des États-Unis dans les affaires intérieures grecques} de Georges Papadopoulos fait l'objet d'interprétations différentes. Pour Le Monde : «il est permis de se demander si Washington n'a pas [...] donné le feu vert à l'opération [...]». Les raisons sont exposées: «le refus d'Athènes de se montrer compréhensif à l'égard de Washington au cours de la crise du Proche- Orient et d'accorder la moindre facilité au ravitaillement d'Israël semble avoir vivement indisposé les protecteurs américains de M.Papadopoulos, ${ }^{59}$ [...] les accords de coopération qui viennent d'être signés avec la Bulgarie ${ }^{60}$. D'ailleurs, pour le journal : « les nouveaux maîtres de la Grèce [...] passent pour de fervents défenseurs de l'OTAN [...]». Le journal cite également les propos d'un général anonyme : «Les Américains n'ont pas fait le coup, mais ils en ont été informés et ont exigé la nomination de l'actuel Premier ministre $[. ..] »^{61}$. Les nouveaux dirigeants de la Grèce sont: "ceux qui viennent d'être installés aux commandes par les chars américains [...] $»^{62}$. En revanche, Le Figaro s'en tient à la version officielle : « Dès le premier jour, le porte- parole du State Department a démenti la moindre intervention de la C I A dans les événements [...] $»^{63}$. Toutefois : «Washington, cette fois-ci n'a pas lieu d'être mécontent du changement [...]. Le Premier ministre a encore plus de raisons d'être apprécié des Américains : il est resté douze ans avocat à Chicago et ne cache pas ses sentiments américanophiles [...] ».

Ainsi, pour les trois journaux, les États-Unis soutiennent le régime des colonels pour des raisons stratégiques : la présence soviétique en Méditerranée et les turbulences au Proche-Orient. Pour Le Monde et L'Humanité, ce soutien participe à la pérennité du régime et entraîne des interventions dans la politique intérieure grecque. Quel est alors le rôle des États-Unis dans la chute du régime survenu en juillet 1974 ? 


\section{4 : Imbroglio chypriote et chute de la dictature}

Depuis 1960, Chypre, ancienne colonie britannique, était une république indépendante dont le statut, défini par les traités de Zurich et de Londres, était garanti par la Grèce, la Turquie et la Grande - Bretagne. Celles-ci y possédaient des bases militaires. Les traités permettaient à ces trois puissances d'intervenir si elles estimaient que leurs intérêts étaient menacés. Chypre était présidée par Mgr Makarios, farouche partisan du «nonalignement » tandis que les pouvoirs étaient répartis en Grecs et Turcs de l'île. Les Grecs constituaient la majorité de la population, soit $4 / 5$ des six cent mille habitants.

31 En 1967, les colonels exprimèrent leur désir de voir Chypre intégrer la Grèce. "Cette union ", en grec Enosis, avait pour conséquence d'opposer le principe des nationalités (des Grecs) à celui du droit des minorités (des Turcs). Mais, surtout, l'Enosis entraînait des tensions entre la Turquie et la Grèce, toutes deux membres de l'OTAN. Mgr Makarios défendait l'indépendance de l'île, position également défendue par l'URSS. En effet, la fin de l'indépendance de l'île signifiait pour celle-ci, l'intégration de Chypre dans l'OTAN dont la Grèce et la Turquie étaient membres. Or, il semble que les ÉtatsUnis aient été soucieux d'étendre leur influence en Méditerranée ${ }^{64}$. L'île connut différentes tensions entre 1967 et 1974.

Parmi celles-ci, en novembre 1967, le général Grivas, farouche partisan de l'Enosis et membre de l'EOKA ${ }^{65}$, responsable de la garde nationale chypriote du président Makarios attaqua deux villages turcs. Cet événement entraîna la mobilisation des troupes turques, de fortes tensions entre les deux communautés de l'île, mais aussi entre la Grèce et la Turquie. Le conflit se résolut par le départ du général Grivas pour Athènes et le retrait des troupes grecques de l'île. L'indépendance de l'île fut réaffirmée. Si Le Monde et L'Humanité estimaient qu'Athènes était « derrière » ce coup de main, Le Figaro soulignait seulement le souhait des colonels de réaliser l'Enosis ${ }^{66}$. Toutefois, la réaffirmation de l'indépendance de l'île semblait marquer la fin de l'Enosis pour les trois quotidiens ${ }^{67}$.Cependant, les tensions demeurèrent entre les deux communautés de l'île, mais aussi entre Athènes et Nicosie ${ }^{68}$.

\section{Le « fiasco » du coup d'État de Nicosie}

Ce coup d'État eut lieu le 15 juillet. Il fut réalisé par la garde chypriote avec l'aide des six cent cinquante officiers grecs qui l'encadraient - officiers dont Mgr Makarios avait demandé le retour à Athènes et au sujet desquels il avait lancé un ultimatum à Athènes fixé au 20 juillet. M. Nicos Sampson - membre de l'EOKA $B^{69}$ - devint président de la République chypriote. La mort de Mgr Makarios fut annoncée par la radio chypriote grecque. Cependant, celui-ci réussit à s'échapper de l'île grâce à l'aide des Britanniques et appela à la résistance à Chypre et au soutien de la communauté internationale.

Pour les trois journaux, le pouvoir d'Athènes est responsable du coup d'État, mais les objectifs semblent différents.

\section{La responsabilité d'Athènes}

Le Monde dénonce ce coup d'État orchestré par Athènes : «[...] la rébellion d'officiers et l'élimination d'un chef de l'État sont bien dans les manières expéditives du pouvoir

Cahiers balkaniques, 38-39 | 2011 
militaire d'Athènes $[. ..] »^{70}$. Pour le journal : il s'agit $d^{\prime}$ : « une tragique mascarade mise en scène de bout en bout par la dictature grecque [...]». L'opposition à travers les propos de Richard Someritis renforce ce point de vue : Pour lui, il s'agit d' : «Un crime signé ", « les assassins de la république libre de Chypre sont les militaires au pouvoir à Athènes [...] les événements ont été fomentés de longue date. $»^{71}$.

Le Monde évoque plusieurs raisons à ce coup d'État. Tout en soulignant que Nicos Sampson est «favorable à l'Enosis ", le journal estime que la raison essentielle est la volonté d'Athènes d'empêcher "la neutralisation" de l'île » ${ }^{72}$ il s'agit donc de se débarrasser de Mgr Makarios. Enfin, il est suggéré, le point de vue n'étant pas développé, un intérêt stratégique des États-Unis: "on attribue aux États-Unis, le souhait de faire de Chypre une dépendance de la Grèce et de la Turquie [...] » ${ }^{73}$.

L'Humanité condamne le coup d'État orchestré par Athènes. Le titre de la page une, le 16 juillet, est très clair: "Chypre: le putsch organisé par les dictateurs grecs". "Enfonçant le clou », L'Humanité reprend les propos de la presse américaine : «le NewYork Times a été obligé d'écrire: "Il ne fait pas de doute que la responsabilité de ce putsch brutal incombe à la sordide dictature grecque $[. ..] \aleph^{74}$. Il s'agit d'une " agression » et d'un « crime » ${ }^{75}$. Si, L'Humanité évoque la volonté d'Athènes de réaliser l'Enosis $^{76}$, pour le journal, les raisons sont uniquement stratégiques : « La junte grecque et ses soutiens atlantiques ne pardonnent pas au gouvernement Makarios, sa politique d'indépendance et son refus de voir Chypre transformée en place d'armes de l'impérialisme en Méditerranée [...] $\gg^{77}$ et le coup d'État répond, à des objectifs géostratégiques américains. : Quand on sait l'intérêt stratégique de l'île, véritable porte-avions en Méditerranée orientale, à proximité des pays du Proche-Orient [...] Quel usage les nouveaux maîtres de Chypre feraient-ils des bases militaires de l'île? Depuis des années, les stratèges de l'OTAN rêvent de pouvoir les employer comme une épée de Damoclès contre le Mouvement national de libération des peuples arabes ${ }^{78}$.

Pour Le Figaro, la responsabilité d'Athènes est également évidente. Il s'agit d'une: " opération coup de poing des généraux politiciens d'Athènes » qui se retrouvent dans «l'embarras [...] » ${ }^{79}$. Car « le plan soigneusement mis au point aurait été compromis lorsqu'il s'est avéré que Mgr Makarios avait survécu [...] son appel à la résistance rendait impossible le déroulement normal de l'action imaginée à Athènes [...] $»^{80}$. L'objectif de ce coup d'État est l'Enosis or: "la communauté turque de l'île ne l'acceptera jamais [...] $»^{81}$. Mais, pour les trois journaux, les États-Unis portent également une part de responsabilité dans ce coup d'État.

\section{Le soutien américain au coup d'État}

39 Si Le Monde rend compte de la position officielle des États-Unis: le maintien de l'indépendance de Chypre, le journal à travers ses éditoriaux dénonce, non pas le rôle, mais plutôt la responsabilité morale des États-Unis dans ce coup d'État : «Les ÉtatsUnis ont montré trop de complaisance au régime grec et à ses excès intérieurs [...] on croit difficilement dans de nombreuses capitales, qui ne sont pas que communistes, au désir proclamé de maintenir la politique de soutien à l'indépendance $[. ..] »^{82}$.

La responsabilité américaine et surtout leur intérêt américain sont dénoncés par l'opposition. Richard Someritis écrit: "Chypre est un formidable porte-avions à quelques kilomètres du Proche-Orient [...] son attachement à la Grèce ou son partage entre la Grèce et la Turquie - solutions auxquelles rêvent les officiers à Athènes - la 
placerait dans le domaine atlantique et par conséquent américain [...] les stratèges de l'OTAN désiraient cette intégration depuis des années (...) leurs alliés exemplaires (le mot est du général Goodpaster ${ }^{83}$ ) d'Athènes viennent de leur ouvrir la voie [...] ». Il en est de même pour Georges Ténékidès. ${ }^{84}$ Pour cet universitaire, la raison ne saurait être l' « Enosis » : « [...] irréalisable [...] [ce serait un] casus belli [...] [avec la Turquie] tout au plus, le gouvernement s'accommoderait - il d'une double annexion c'est-à-dire du démembrement de l'île [...] un autre objectif, celui-ci non avoué [mais] certain [...] celui de la substitution à la politique d'indépendance et de non-alignement [à] une politique de dépendance [...] [et qui] amarrerait la totalité de l'île à une organisation militaire [...] ». Enfin, plus que le rôle, il semble que les États-Unis soient "derrière " ce coup d'État pour Claude Bourdet ${ }^{85}:$ « [...] Si Sampson et les putschistes gagnent, lîle rentrera dans l'OTAN et M. Kissinger aura sa base [...] un pas de plus sera fait vers l'américanisation de la Méditerranée orientale [...] ». Mais, très vite, le journal dénonce l'attitude américaine, car les États-Unis, après avoir défendu le maintien de l'indépendance de l'île, se déclarèrent très rapidement pour son partage. ${ }^{86}$ Pour le journal : "c'est accepter l'inacceptable [...] moins neutraliste Chypre sera un point d'appui plus sûr pour la VI flotte ». C'est le : «triomphe de la " real politik » ».

41 Pour Le Figaro : «Washington était de toute évidence au courant de ce qui se préparait à Nicosie. $»^{87}$. Le journal dénonce l'attitude équivoque des États-Unis: «Entre les principes dont se réclament officiellement les grandes puissances et les actes qu'elles sont prêtes à accomplir [...] il y a une sérieuse marge de manoeuvre qui peut-être fatale aux petits États [...]. L'administration américaine a plus besoin en Méditerranée orientale [...] de la coopération active du gouvernement d'Athènes que du retour à Nicosie de [Mgr] Makarios [...] $»^{88}$. Le Figaro n'évoque pas la volonté américaine d'installer des bases à Chypre. Toutefois, il indique : « [...] le seul intérêt de l'URSS dans l'immédiat est de rétablir la neutralité dans l'île. La seule crainte est de voir Chypre devenir une base militaire atlantiste [...] il y aurait un déséquilibre certain, maintenant que l'URSS comme les États unis, considère la Méditerranée comme sa chasse gardée (...) $»^{89}$.

42 Pour L'Humanité, les États-Unis ont joué un rôle et sont responsables du coup d'État. Ceci est lié à l'objectif de celui-ci : l'insertion de Chypre dans le dispositif américain de l'OTAN : «Quand on connaît l'influence prépondérante dont [...] les États-Unis jouissent à Athènes et, quand on sait l'intérêt stratégique de l'île [...] il est bien difficile d'imaginer que le coup de Nicosie ait pu être entrepris à l'insu des Américains [.... ${ }^{90}$. "Comme l'écrit le "New York Times", « depuis des années l'administration Nixon considérait l'archevêque Makarios comme le "Castro de la Méditerranée » [...] Quiconque refuse l'alignement sur les États-Unis est donc un « rouge » [...] $»^{91}$.

43 Les trois journaux s'inquiètent de la situation créée par le coup d'État et soulignent les risques de tension liés à celui-ci.

\section{Risque de conflit international et débarquement turc}

Les risques sont unanimement dénoncés. Ce coup d'État crée des problèmes à plusieurs échelles. La première échelle est celle de l'île. Le coup d'État peut créer un conflit au sein de la communauté grecque de l'île. Le Figaro souligne le risque de guerre civile, entre les Grecs de l'île, entraîné par l'appel à la résistance de Mgr Makarios ${ }^{92}$. De plus, ce coup d'État entraîne des problèmes entre les communautés grecque et turque de l'île qui «vivent dans un état de conflit permanent " ". Mais il risque aussi d'entraîner un 
conflit entre Athènes et Ankara. Comme les deux autres journaux, L'Humanité précise que : «Les forces armées turques [sont] en état d'alerte $»^{94}$.

Ce coup d'État déstabilise aussi la stratégie de l'OTAN en Méditerranée orientale : « Les trois piliers du dispositif de l'OTAN, Grèce, Turquie et Chypre sont ébranlés. Deux États membres de l'Alliance atlantique risquent de [...] se trouver en conflit ouvert [...] ce qui remettrait en cause toute la cohésion stratégique élaborée sous la houlette des Américains [...] $»^{95}$.

Enfin, il risque de provoquer un conflit ou du moins des tensions internationales. Pour Le Monde : «On peut donc s'attendre à l'explosion d'une crise majeure en Méditerranée orientale [...] à [...] un affrontement entre les Américains [...] et les Soviétiques $»^{96}$. Le Figaro explique ces tensions : "Moscou acceptera difficilement que l'île [...] perde son indépendance, d'autant que la Méditerranée orientale va reprendre toute son importance avec la réouverture prévisible du canal de Suez ${ }^{97}[. .$.$] la crise de Chypre$ risque de montrer les limites de la détente entre les deux Grands [...] ${ }^{98}$.

Les trois journaux rendent compte de l'opposition internationale suscitée par ce coup d'État: «le ton général est celui d'une dénonciation du coup de force, personne ne prenant le parti du gouvernement d'Athènes " ${ }^{99}$. L'Humanité précise : " même l'OTAN demande le retrait des officiers grecs $»^{100}$. Le Monde et L'Humanité soulignent d'ailleurs l'isolement de la junte : «Même aux États-Unis, dont on connaît la sympathie pour les colonels grecs, le gouvernement ne cache pas son embarras [...] $»^{101}$ « Le gouvernement d'Athènes est soumis à de multiples pressions et interventions [...] $»^{102}$.

Le 20 juillet 1974, les troupes turques débarquèrent au nord de Chypre. La mobilisation générale fut décrétée à Athènes. Le 22 juillet, un cessez-le-feu intervint entre les Turcs et les Grecs à Chypre. Pour les trois journaux, le débarquement turc relève de la seule responsabilité américaine. Le Monde juge sévèrement l'attitude des États-Unis, il s'agit d': « un effroyable gâchis qui [marque] les inconséquences et les arrières pensées des grandes puissances $[. ..] »^{103}$. «Cher Henry » [...] de toute évidence sa diplomatie n'a pas cru en une riposte turque $[. ..] »^{104}$.

Comme Le Monde, Le Figaro souligne les responsabilités internationales dans ce débarquement : "les grandes puissances qui ont toléré, puis pratiquement entériné le putsch, sont responsables de l'intervention turque [...] $»^{105}$. Mais, pour Le Figaro, les États-Unis ont fait preuve d'inconséquence : «Toute l'action des États-Unis [...] visait à dissuader la Turquie d'intervenir militairement tout en ne disant rien ou en ne faisant rien contre la junte militaire grecque». Pour Le Figaro, Henry Kissinger est responsable : «En jouant Athènes contre Ankara [...] en se refusant à prendre tout de suite le parti de Mgr Makarios. [...] M Kissinger - c'est évident - ne croyait pas à l'éventualité d'une intervention militaire turque $[. ..] »^{106}$. Le ton et l'explication sont les mêmes dans L'Humanité: " Les États-Unis portent, dans la sanglante affaire de Chypre, une écrasante responsabilité : même la presse de tonalité «atlantique » le reconnaît avec mélancolie [...] l'éditorialiste du Figaro gémit devant cet « effroyable gâchis [...] ${ }^{107}$. Pour le journal : « Le conflit chypriote met en lumière la conspiration permanente que mènent les puissances impérialistes au premier chef - les États-Unis - pour s'assurer le contrôle des points stratégiques de la planète [...]. Ce n'est pas le gouvernement soviétique, mais le gouvernement américain qui appuie les régimes fascistes ${ }^{108}$.

L'isolement international de la junte d'Athènes - consécutif à la condamnation internationale et qui résultait du coup d'État de Nicosie - constitue pour les trois 
journaux, une menace pour le régime d'Athènes, menace dont ils rendent compte en évoquant des rumeurs de coup d'État.

\section{La chute du régime : une décision américaine ?}

51 Avant la chute du régime des colonels, les trois journaux font état de rumeurs qui circulaient à Athènes. Ces rumeurs concernaient un éventuel changement de régime et étaient consécutives aux déclarations du secrétaire d'État, Henry Kissinger.

Le Monde reprend ses déclarations, faites le 22 juillet à Washington, dans un éditorial intitulé «Dear Henry»: "nous ne pouvons pas formuler un jugement clair sur les forces qui vont d'emparer du gouvernement. ». Ces propos appellent le commentaire suivant: «volontiers désinvolte avec ses alliés [...], mais jamais encore le chef de la diplomatie américaine n'avait annoncé un coup d'état dans une capitale amie [...] il vient de le faire implicitement [...] ». En conséquence, pour le journal : « on sait que la forme actuelle du pouvoir va changer [...] on parlait beaucoup du retour du roi [...] [de] M. Caramanlis $"^{109}$.

Quant au Figaro, si le journal précise : : «le régime du général Ghizikis a été fortement ébranlé par la défaite [...] on parlait également du retour de l'ancien Premier ministre Constantin Caramanlis [...]». Les propos d'Henry Kissinger sont jugés très sévèrement : "Washington «lâche » les colonels » Kissinger renvoie Machiavel chez les auteurs de la bibliothèque rose [...] virtuose de la diplomatie [...] [il dénonce] les responsables grecs d'une opération malencontreuse, sur laquelle il a pourtant lui-même fermé les yeux pendant cinq jours, et qu'il n'aurait certainement pas désavouée, si elle avait réussi. [...]. Étrange comportement [...] à l'égard d'un gouvernement allié! M. Kissinger ne s'embarrasse pas de tels scrupules ! [...] $»^{110}$.

Quant à L'Humanité, les déclarations du secrétaire d'État confirment la thèse que le journal défend depuis avril 1967, à savoir : les liens entre les États-Unis et la dictature : « N'est-ce pas M. Kissinger qui a annoncé hier en premier [...] qu'un changement allait intervenir dans la composition du " gouvernement » grec ? On ne saurait mieux avouer l'existence des liens très particuliers qui relient comme un cordon ombilical les colonels d'Athènes à la Maison-Blanche $[. ..] »^{111}$

\section{Le retour de Constantin Caramanlis : un événement imprévu}

Pour les trois quotidiens, ce retour constitue une véritable surprise.

Pour Le Monde : « le choc a été brutal [...] on s'attendait [...] à des luttes intestines dans l'armée, à un nouveau coup d'État [...]. À 19 h 15 [...], la radio annonçait que la junte capitulait inconditionnellement, le pouvoir était rendu aux civils, les colonels, les généraux, les tortionnaires déclaraient forfait [...]». Mais pour Le Monde, Constantin Caramanlis ne l'avait pas prévu lui-même «nous recevant à Paris, le 10 juillet [...] M. Caramanlis exprimait son pessimisme sur les possibilités d'un changement radical [...] $»^{112}$. Pour Le Figaro: "Les généraux grecs rappellent M. Caramanlis $»^{113}$. Enfin, dans L'Humanité : « c'est tout le contraire de ce que [les militaires] avaient prévu qui se produit. C'est leur régime qui s'effondre. [...] $»^{114}$. Toutefois, la portée, le sens de cet événement ne sont pas le même pour les trois quotidiens.

Comme Le Monde, L'Humanité juge que le retour de M. Caramanlis marque la fin de la dictature en Grèce : «La dictature s'effondre en Grèce ». "Après sept années d'une 
dictature militaire implacable et anachronique, une page de l'histoire de la Grèce vient d'être tournée [...] l'essentiel - ce qui fait l'unanimité - c'est la chute des " colonels » $[. ..] . »^{115}$. Pour Le Figaro, il s'agit de la formation d'un gouvernement civil qui doit régler le problème chypriote, ce que les militaires au pouvoir sont incapables de faire: «ce recul des militaires donne [...] une idée du désarroi dans lequel ils se trouvent $[. ..] »^{116}$. D'ailleurs et à la différence des deux autres journaux, Le Figaro n'évoque pas « la chute de la dictature », mais évoque «la fin du régime des colonels» et la fin du «régime militaire $[. .]. »{ }^{117}$

Le retour de M. Caramanlis et la chute de la dictature sont pour les trois journaux directement liés au coup d'État manqué de Nicosie. Il s'agit d'une conséquence intérieure de ce coup d'État. Celui-ci aurait entraîné une opposition interne, une opposition internationale et l'isolement de la junte.

Les trois journaux évoquent l'opposition intérieure. Cette opposition venait de l'armée elle-même et plus particulièrement des forces stationnées dans le nord du pays: «[Elles] ont joué un rôle essentiel [...] c'est d'elles que parvenait à la veille du bouleversement un manifeste dénonçant la "politique criminelle» de la junte et prônant le rappel du dirigeant de droite exilé [...] " ${ }^{118}$. Cette idée est également reprise par L'Humanité et Le Figaro: "On sait maintenant que c'est de Salonique qu'est partie l'idée $\mathrm{du}$ 《 juste retour dans les casernes $»^{119}$.

Mais Le Monde reprend aussi « la thèse » de Constantin Caramanlis, présentée le 10 juillet 1974, dans une interview, pour expliquer la chute du régime. Pour le futur Premier ministre, le pouvoir en Grèce reposait sur trois piliers : «la junte (...) l'armée dans toutes ses composantes et les Américains (...) » ${ }^{120}$. Pour le journal : « l'hypothèse la plus répandue ici est le fait que les trois piliers du régime (...) se sont effondrés sous l'effet du « séisme chypriote ${ }^{121}$. En effet, pour le journal: «la junte n'était plus en mesure de mener des négociations ni «de livrer une guerre victorieuse à la Turquie (...) ». De plus «l'humiliation de la défaite (...) provoqua l'éclatement des liens de solidarité qui liaient auparavant les divers clans de l'armée (...)».

61 L'Humanité est en revanche le seul journal à évoquer une "résistance intérieure » pour expliquer la chute du régime. Il s'agit des propos de Grégoris Farakos, membre du bureau politique du Parti communiste grec: "ce grand succès est devenu possible grâce à la lutte du peuple grec, et particulièrement de ses jeunes (...) au cours des manifestations de l'École Polytechnique en novembre dernier [...] $»^{122}$. Il est cependant « logique » de retrouver cette thèse dans le journal. En effet, le Parti communiste grec, le Parti communiste français et le journal ont toujours défendu la thèse d'une opposition populaire et unanime à la junte ainsi que celle de l'isolement international du régime. Mais plus que l'opposition intérieure, il semble que les États-Unis aient joué un rôle essentiel dans la chute du régime.

\section{Le rôle des États-Unis}

Les trois journaux estiment que ce pays a «lâché » les militaires grecs en raison de l'échec du coup d'État de Nicosie.

63 Tout en évoquant l'influence américaine, Le Monde évoque aussi rôle d'Ankara dans l'évolution politique grecque : "c'est à l'ennemi héréditaire que les Grecs doivent le retour à la démocratie [...], Ankara (...) aurait exigé de négocier à Genève avec un gouvernement véritablement représentatif $[. ..] »^{123}$. Quant aux Américains: "ces 
derniers [...] étaient mécontents des performances de l'équipe du général Ghizikis [...] des incapables selon l'ambassadeur des États-Unis [...] craignant une passation de pouvoir dans l'anarchie, les États-Unis auraient dès lors décidé de "lâcher " leurs protégés en les incitant à livrer le pouvoir à une coalition d'hommes politiques conservateurs $[. ..] »^{124}$. Toutefois la position du journal semble infirmer par les propos de M. Mavros, nouveau ministre des Affaires étrangères grecques, interviewé le 26 juillet. À la question: "Pensez-vous que les Américains ont exercé des pressions ? ", «M. Mavros sourit et répond : "N’est-ce pas M. Kissinger qui a annoncé le premier le changement qui allait se produire à Athènes? ». Ces propos ne laissent aucune ambiguïté sur le rôle des États-Unis dans la chute de la dictature.

Pour Le Figaro, les choses sont claires : «M. Caramanlis, malgré sa popularité auprès de l'opinion publique rentre d'une certaine manière à Athènes dans les fourgons de l'étranger $[. ..] »^{125}$. Les États-Unis ont lâché la Grèce qui a été traitée comme : "une colonie américaine $»^{126}$. Le responsable est Henry Kissinger qui a fait preuve de " cynisme et [d]'efficacité $~^{127}$. " [...] Il a vraisemblablement suffi d'un claquement de doigts des hommes de la CIA ou de l'ambassade des États-Unis (...) ». Le journal souligne ainsi l'absence totale de maîtrise des Grecs sur leur destin politique. Le journal exprime clairement l'idée que les États-Unis sont les véritables instigateurs de tous les changements politiques en Grèce: «M. $\operatorname{Sisco}^{128}[. .$.$] dans son rapport à Washington$ préconisait trois changements : la nomination de M. Cléridès à Chypre, le retour de Mgr Makarios, mais seulement en tant que chef d'Église, l'arrivée d'un gouvernement civil à Athènes. Avec le coup de pouce des Turcs, l'essentiel est accompli [...] $»^{129}$. Cette position constitue donc une évolution de taille dans le discours du Figaro qui jusque-là niait toute intervention directe des États-Unis dans la politique grecque.

Pour L'Humanité, la chute de la dictature relève d'une décision américaine. Revenant sur les déclarations d'Henry Kissinger du 22 juillet, le journal publie: «C'était reconnaître du même coup que la junte militaire d'Athènes n'existait que par la grâce de Washington. Que l'impérialisme américain et ses alliés atlantistes doivent donc être tenus pour responsables des terribles épreuves infligées au peuple grec depuis plus de sept ans [...] l'échec du coup d'État de Chypre [...] ont donc, enfin contraint les ÉtatsUnis à changer quelque chose au régime de terreur instauré en Grèce par les soins de leur CIA, et en application du plan de l'OTAN [...] Les colonels [ont été] mis en demeure de charger M. Caramanlis de former un nouveau gouvernement $[. ..] »^{130}$. Le journal réitère ces propos le lendemain : « Les dictateurs d'Athènes n'étaient pas seulement des assassins. Ils ont administré la preuve qu'ils étaient des imbéciles et leur faillite, sur tous les plans, a contraint Washington, la mort dans l'âme, à leur retirer sa protection $[. ..] »^{131}$.

66 L'échec du coup d'État de Nicosie orchestré par les "colonels» et soutenu par les Américains apparaît comme la cause essentielle de la chute du régime d'après les trois quotidiens. Ce coup d'État raté aurait entraîné l'intervention turque, l'isolement de la junte et l'abandon de celle-ci par les États-Unis. Ainsi, la question des intérêts géostratégiques américains en Méditerranée orientale durant la dictature des colonels, malgré les divergences de points de vue ou les nuances d'appréciations exprimées par les trois quotidiens nationaux français, montre le poids des États-Unis et de la guerre froide dans l'évolution politique grecque. La Grèce et les Grecs ne semblent pas, durant cette période, maîtres de leur destin! 


\section{NOTES}

1. Sur le sujet: Woodhouse Christopher- Montague, The rise and the fall of the Greek colonels. Londres. Granada. 1985. Athenes-Presse-Libre, Toute la Grèce, Paris. 1974.

2. ASPIDA, terme grec signifiant «bouclier». Il s'agit en fait d'un acronyme qui signifie : «Officiers, sauveurs de la patrie, des idéaux et de la démocratie ».

3. Cette situation politique ne manque pas de provoquer de vives réactions de la part du Monde et de L'Humanité qui estiment que si la Grèce sort de la crise politique, la nomination de M. Canellopoulos est totalement illégitime. Pour les deux journaux, la droite minoritaire ne doit ni diriger le gouvernement, ni préparer les élections.

4. L'Humanité, le 23 avril 1967, p. 3.

5. L'Humanité, le 15 avril 1968, p. 3.

6. Le Monde, le 26 avril 1967, p. 2.

7. Ibid.

8. Le Monde, le 22 avril 1967, p. 1.

9. Éditorial, le 22 avril 1967, p. 1.

10. L'Humanité, le 23 avril 1967, p. 3.

11. Éditorial, le 22 avril 1967, p. 1.

12. Le Figaro, le 11 mai 1967, p. 3.

13. Le Figaro, le 7 septembre 1967, p. 5 et le 8 septembre 1967, p. 5.

14. Ibid.

15. Ibid.

16. Le Figaro, le 21 avril 1971, p. 4.

17. Le Figaro, le 17 août 1972, p.12. L'individu est présenté comme: « un jeune homme qui travaillait auparavant à la radio... "

18. Le Figaro, le 20 avril 1967, p. 3.

19. Le Figaro, le 26 avril 1967, p. 9.

20. L'Humanité, le 22 avril 1967, p. 3.

21. L'Humanité, le 25 avril 1967, p. 2.

22. L'Humanité, le 27 avril 1967, p. 2.

23. L'Humanité, le 4 mai 1967 , p. 3.

24. Jacques Duclos, membre du comité central du PCF.

25. L'Humanité, le 12 septembre 1967, p. 1.

26. Le Monde, 2-3 avril 1967, p. 4.

27. Le Monde, le 21-22/04/1968, p. 5.

28. Le Monde, 26 avril 1967, p. 1.

29. Le Monde, le 27 avril 1967, p. 2.

30. Le Monde, 24 avril 1971, p. 7. Journaliste grec. Fondateur à Paris du bulletin « Athènes- PresseLibre» en octobre 1967. Ce bulletin fut une des principales sources d'information sur les événements grecs, des principaux médias français incluant les trois journaux étudiés. Co-auteur du Livre noir de la dictature en Grèce, Paris. Seuil, Collection Combat, 1969.

31. Le Figaro, le 15 décembre 1967, p. 4.

32. L'Humanité, le 15 décembre 1967, p. 1. Le roi lança son appel à la rébellion de Larissa, une escadre située à proximité d'une base de l'OTAN.

33. Le Monde, 14-15 décembre 1969, p. 2.

34. Le Monde, le 20 avril 1968,p. 4.

35. Le Monde, le 22 avril 1972, p. 10.

36. Le Monde, 14-15 novembre 1971, p. 5. 
37. Le Monde, le 22 avril 1972, p. 10.

38. Le Monde, le 14-15 décembre 1969, p. 2.

39. L'Humanité, le 15 décembre 1969, p. 2.

40. L'Humanité, le 15 décembre 1969, p. 2.

41. L'Humanité, le 21 avril 1971, p. 2.

42. Le Figaro, le 11 décembre 1969, p. 13.

43. La Roumanie était officiellement intégrée au bloc soviétique, mais M. Ceaucescu souhaitait avoir une politique étrangère plus ouverte vers l'Occident que celle suivie par le bloc soviétique. Quant à la Yougoslavie du maréchal Tito, elle appartenait aux pays « non alignés ».

44. Richard Nixon, républicain et anticommuniste.

45. En 1969, le colonel Kadhafi fit un coup d'État qui entraîna l'éviction des États-Unis du pays.

46. Le Figaro, le 11 décembre 1969, p. 13.

47. Le Figaro, le 20-21 septembre 1969, p. 5.

48. Le Figaro, le 20-21 septembre 1969, p. 5.

49. Le Monde, le 20 avril 1968, pp. 1 et 4.

50. Litton : entreprise américaine d'investissement.

51. Le Monde, le 20 avril 1968, p. 4.

52. Le Monde, le 21-22 avril 1968, p. 5. Mario Ploritis était un écrivain grec réfugié en France.

53. L'Humanité, le 21 avril 1971 p. 2.

54. Georges Papadopoulos cumula rapidement tous les pouvoirs. En décembre 1967, il prit le titre de Premier ministre et de ministre de l'Intérieur ; en décembre 1968, il fut également ministre de la Défense. Il renforça ses pouvoirs militaires en décembre 1969 et devint président de la République en juillet 1973.

55. Sur la mise en place de la République : les trois journaux contestent la thèse officielle du complot dans la marine. En revanche, ils expliquent ce changement institutionnel par une opposition multiple et croissante : opposition du roi, de l'armée et de la population en raison de difficultés économiques.

56. Le Monde, le 5 juin 1973, p. 4.

57. Le Monde, 15 juin 1973, p. 1.

58. L'Humanité, le 9 juin 1973, p. 2.

59. Le régime opéra une politique de rapprochement avec les pays de l'Est. Lors de la guerre du Kippour, il semble qu'il n'autorisa pas les avions israéliens à se ravitailler sur les bases grecques.

60. Le Monde, le 27 novembre 1973, p. 1.

61. Le Monde, le 30 novembre 1973, p. 3.

62. L'Humanité, le 27 novembre 1973, p. 3.

63. Le Figaro, le 30 novembre 1973, p. 5.

64. Cf. Asmussen, Jan., Cyprus at war: diplomacy and conflict during the 1974 crisis. London; NewYork. I.B. Tauris. 2008. $364 \mathrm{p}$.

Carandon, Lord, Frazee, Charles et al., Greece and Cyprus in history. Amsterdam: A ;M. Hakkert, 1985. $227 \mathrm{p}$.

Hitchens, Christopher, Cyprus. London: Melbourne ; New-York. Quartet Book, 1984, 192 p.

Koumoulides, John T.A., Cyprus in transition, 1960-1985. London: Trigraph, 1986.173 p.

Le différend gréco-turc. Colloque du centre d'études et de recherches internationales de la fondation nationale des sciences politiques. 29-30 mai 1986. Sous la direction de Semih Vaner, Paris : L'harmattan,1988.284 p.

Miller, James Edward., The United States and the making of modern Greece: history and power, 19501974. Chapel Hill (N. C): University of North Carolina press. 2009, 301 p.

Necatigil, Zaim M., The Cyprus question and the Turkish position in international law. Second edition, Oxford: Oxford University,1993. $482 \mathrm{p}$

Panteli, Stavro., A new history of Cyprus. From the earliest times to the present day. London: East-West 
Publication, 1984. $437 \mathrm{p}$.

Polyviou G., Cyprus : conflict and negotiation, I. New York. Holmes and Meier Publication. 1980.

$246 \mathrm{p}$.

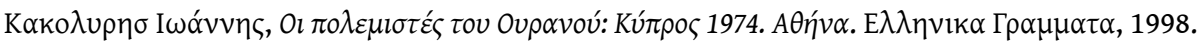

65. EOKA organisation de résistance contre les Britanniques et qui prônait l'Enosis dès l'indépendance.

66. Le Monde, le 18 nov. 1967, p. 1, Le Figaro, le 27 novembre 1967, p. 1, L'Humanité, le 22 novembre 1967, p. 1.

67. Voir Le Figaro, le 29 novembre 1967, p. 3, L'Humanité, Le $1^{\mathrm{er}}$ décembre 1967, p. 1 et Le Monde, 29 novembre 1967, p. 1. Le Figaro le 10 novembre 1967, p. 5.

68. En exemple, en mars 1970, Mgr Makarios échappa à un attentat. En juillet 1970, des élections eurent lieu à Chypre qui sanctionnèrent les partisans de l'Enosis. Malgré cela, en 1971, un comité de coordination de lutte pour l'Enosis fut créé à Chypre. En février 73, les partisans de l'Enosis multiplièrent les attentats. Mgr Makarios fut réélu président. Le général Grivas mourut en janvier 1974. Mais, en juin, Mgr Makarios accusa la garde nationale grecque de Chypre de comploter contre lui et il demanda leur renvoi le 5 juillet.

69. L'EOKA B est l'organisation qui prit la suite de l'EOKA après l'indépendance de Chypre.

70. Le Monde, le 16 juillet 1974, p. 1. Le journal crut d'abord à la mort de Mgr Makarios.

71. Le Monde, le 16 juillet 1974, p. 2.

72. Le Monde, le 16 juillet 1974, p. 1.

73. Le Monde, le 16 juillet 1974, p. 2.

74. L'Humanité, le 17 juillet 1974, p. 2.

75. L'Humanité, le 16 juillet 1974, p. 1.

76. L'Humanité, le 20 juillet 1974 , p. 2.

77. L'Humanité, le 17 juillet 1974, p. 1.

78. L'Humanité, le 16 juillet 1974 , p. 1.

79. Le Figaro, le 16 juillet 1974, p. 2.

80. Le Figaro, le 17 juillet 1974, p. 2.

81. Le Figaro, le 16 juillet 1974, p. 1.

82. Le Monde, le 17 juillet 1974, p. 1.

83. Le général Goodpaster, américain, était l'ancien responsable militaire américain au Vietnam et le nouveau commandant des forces de l'OTAN en Europe.

84. Georges Ténékidès est présenté par le journal comme professeur associé à la faculté de droit, d'économie et de sciences sociales de Paris. Le Monde, le 19 juillet 1974, p. 2.

85. Le Monde, le 20 juillet 1974, p. 2. Claude Bourdet est un ancien résistant parisien.

86. Déclaration de Washington, le 20 juillet 1974, p. 2.

87. Le Figaro, le 22 juillet 1974, p. 1.

88. Le Figaro, le 18 juillet 1974, p. 2.

89. Le Figaro, le 22 juillet 1974, p. 2 ..

90. L'Humanité, le 16 juillet 1974, p. 1.

91. L'Humanité, le 20 juillet 1974, p. 1.

92. Le Figaro, le 17 juillet 1974, p. 2.

93. Le Figaro, le 16 juillet 1974, p. 1.

94. L'Humanité, le 16 juillet 1974, p. 2.

95. Le Figaro, le 16 juillet 1974, p. 1.

96. Le Monde, le 17 juillet 1974, p. 1.

97. Le canal de Suez principal point de passage mondial du pétrole avait été fermé à la suite du conflit israélo-arabe de 1973. Rappelons que l'Égypte se trouvait sous influence des États-Unis depuis 1972 et Chypre se situe à quelques centaines de milles marins du canal.

98. Le Figaro, le 16 juillet 1974, p. 1. 
99. Le Monde, le 17 juillet 1974, p. 2.

100. L'Humanité le 17 juillet 1974, p. 1 et le 18 juillet 1974, p. 2.

101. L'Humanité, le 17 juillet 1974, p. 2.

102. Le Monde, 19 juillet 1974, p. 2.

103. Le Monde, le 23 juillet 1974, p. 1.

104. Le Monde, le 24 juillet 1974, p. 1.

105. Le Figaro, le 22 juillet, p. 1.

106. Le Figaro, le 22 juillet 1974, p. 2.

107. L'Humanité, le 23 juillet, page 1.

108. L'Humanité, le 23 juillet, p. 1.

109. Le Monde, le 24 juillet 1974, p. 1.

110. Le Figaro, le 23 juillet 1974, p. 3.

111. L'Humanité, le 24 juillet 1974, p. 1.

112. Le Monde, le 25 juillet 1974, pp. 1 et 3.

113. Le Figaro, 24 juillet 1974, p. 1.

114. L'Humanité, le 24 juillet 1974, p. 1.

115. L'Humanité, le 25 juillet 1974, p 1.

116. Le Figaro, le 24 juillet 1974, p. 2.

117. Le Figaro, le 24 juillet 1974, p. 2.

118. Ce manifeste est publié par Le Monde, le 25 juillet en page trois. Son titre : «Deux cent cinquante officiers demandent le retour de M. Caramanlis ». Il émane du troisième corps d'armée et a été rédigé le 21 juillet. Dans celui-ci les officiers exposent leur revendication. Il souhaite le retour de M. Caramanlis, car celui-ci «bénéficie de la confiance de la majorité du peuple et de l'armée ». Ils demandent le règlement immédiat de la crise chypriote "sur la base du statut constitutionnel en vigueur avant le coup d'État du 15 juillet». Ils demandent également «la convocation dans les six mois d'élections libres ». Ils dénoncent la politique du régime en place et le soutien accordé à celui-ci par les États-Unis : « ceux qui ont le pouvoir... leurs actes criminels nous ont conduits au bord de la catastrophe nationale... nous invitons [les États-Unis] a cesser sans délai l'appui qu'ils accordent depuis sept ans au groupe gouvernant à Athènes... et [à] ne pas intervenir dans nos affaires intérieures... ».

119. Le Figaro, le 25 juillet 1974, p. 2.

120. Le Monde, le 25 juillet 1974, p. 3.

121. Le Monde, le 25 juillet 1974, p. 3.

122. L'Humanité, le 29 juillet 1974, p. 2.

123. Le Monde, le 25 juillet 1974, p. 1.

124. Le Monde, le 25 juillet 1974, p. 3.

125. Le Figaro, le 24 juillet 1974, p. 2.

126. Le Figaro, le 24 juillet 1974, p. 2.

127. Le Figaro, le 24 juillet 1974, p. 2.

128. M. Sisco était le sous- secrétaire d'État américain aux Affaires étrangères responsable du Moyen-Orient.

129. Le Figaro, le 25 juillet 1974, p. 4.

130. L'Humanité, le 24 juillet 1974, p. 1.

131. L'Humanité, le 25 juillet 1974, p. 1. 


\section{RÉSUMÉS}

L'analyse de la dictature des colonels grecs (1967-74) vue par 3 quotidiens français révèle, par ses divergences, les orientations de la presse française.

Il s'agit de présenter dans cet article, les enjeux géostratégiques de la dictature des colonels en Grèce, de montrer si ce régime, en Méditerranée, durant la guerre froide, présentait un intérêt pour les États-Unis, et ceci, à travers les analyses de trois quotidiens nationaux français : Le Monde, Le Figaro et L'Humanité. Cet article s'articule autour de trois périodes. La première, 1967, présente la thèse officielle du régime : la mise en place de la dictature en raison d'une menace communiste imminente. Cette partie montre la divergence d'analyse des trois journaux sur cette thèse, mais aussi, sur le rôle des États-Unis. La deuxième période se concentre sur les années 1968 à 1973 et montre les points de vue sur l'existence d'une protection américaine du régime. Si pour les trois journaux, les États-Unis soutiennent le régime pour des raisons géostratégiques, en revanche, pour Le Monde et l'Humanité, cette protection va au-delà, car elle permet au régime de se maintenir. Enfin, la troisième partie traite de l'année 1974, du coup d'État manqué à Chypre, de la chute de la dictature, et là encore, du rôle, voire de la responsabilité des États-Unis. Ces divergences, convergences ou nuances d'appréciations constituent une somme d'interrogation et de recherches.

The purpose of this article is to present the geostrategical stakes of the colonels' dictatorship in Greece, through the analysis of three French national daily newspaper: Le Monde, L'Humanité and Le Figaro. It will show whether or not this regime, in the Mediterranean, during the cold war, represented an interest for the US. This article turns around three periods. The first, 1967, presents the regime's official standpoint: the establishment of the dictatorship because of the imminent communist threat. This part shows the divergence of the three newspapers analysis on this standpoint but also on the role of the US. The 2 nd period concentrates on the years 1968 to 1973 and exposes the points of view that the American protected the regimeIf for the three newspapers, the US supported the regime for geostrategical reasons, on the other hand, for Le Monde and L'Humanité, this protection goes far beyond this. Finally, the third part covers 1974, the failed coup d'état in Cyprus, the fall of the dictatorship and there again the role or even the responsibility of the US. These divergence, convergence or the different French nuances always argued in the sources are today a basis of questioning and research.

\section{INDEX}

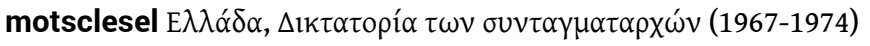

Mots-clés : diplomatie américaine (1967-1974), presse française, géostratégie en Grèce

Index chronologique : dictature des colonels (1967-1974)

Thèmes : Histoire

Keywords : french press, american diplomacy (1967-74), greek colonels dictatorship (1967-1974), geostrategy in Greece, coup d'etat, History, Greece, Turkey, United States

motsclestr Yunanistan, Albaylar diktatörlük (1967-1974)

motsclesmk ГРцИЈА

Index géographique : Grèce 